\title{
Analyse des services d'eau non conventionnels dans les quartiers à habitat spontané : cas du quartier EBA'A dans la périphérie de la ville de Yaoundé (région du centre au Cameroun) \author{
habitat districts: case study of the EBA'A District in the \\ periphery of the Younde City (Center region in Cameroon)
} \\ Analysis of non-conventional water services in spontaneous
}

\author{
Barthelémy Ndongo, Emmanuel Ngnikam et Stéphane Lako Mbouendeu
}

Volume 25, numéro 2, 2012

Reçu le 18 octobre 2010, accepté le 21 juin 2012

URI : https://id.erudit.org/iderudit/1011605ar

DOI : https://doi.org/10.7202/1011605ar

Aller au sommaire du numéro

\section{Éditeur(s)}

Université du Québec - INRS-Eau, Terre et Environnement (INRS-ETE)

\section{ISSN}

1718-8598 (numérique)

Découvrir la revue

\section{Citer cet article}

Ndongo, B., Ngnikam, E. \& Mbouendeu, S. L. (2012). Analyse des services d'eau non conventionnels dans les quartiers à habitat spontané : cas du quartier EBA'A dans la périphérie de la ville de Yaoundé (région du centre au Cameroun). Revue des sciences de l'eau / Journal of Water Science, 25(2), 153-163. https://doi.org/10.7202/1011605ar

\section{Résumé de l'article}

Cet article présente une analyse du service d'eau non conventionnel (service n'ayant fait l'objet d'aucune convention (cahier de charge, contrat, norme ou règlement, etc.), et donc organisé de façon intuitive par les utilisateurs) d'un quartier à habitat spontané de la périphérie de Yaoundé (EBA'A). L'étude aura consisté à : identifier les caractéristiques de ce service, relever les contraintes auxquelles ses usagers sont soumis, identifier les principaux déterminants de satisfaction de la clientèle et définir les perspectives d'amélioration de ce service. Pour cela, une enquête ménage a été effectuée dans le quartier sur un échantillon représentatif de la population étudiée. Cette enquête fut complétée par des observations et mesures faites sur le terrain. L'analyse des données collectées révèle que les services d'eau locaux non conventionnels s'appuient sur des ouvrages de desserte autonomes qui sont considérablement éloignés des usagers. Ce type de service semble contraignant pour les ménages d'EBA'A, et ce, plus sur le plan des conditions d'approvisionnement que du coût du service. C'est pourquoi, en guise d'amélioration, les ménages souhaitent avoir un service d'eau potable proche et permanent, même si son coût demeure élevé. Cependant, il existe un seuil de $2,10 \$ \bullet \mathrm{m}^{-3}$ pour ces populations. Dans ces conditions, un délai de paiement des factures d'au moins dix jours, avec des interruptions de service n'excédant pas trois jours, semble tolérable pour ces ménages. Ainsi, les services d'eau potable non conventionnels pourraient s'avérer être des alternatives d'accès à l'eau potable satisfaisantes dans les quartiers défavorisés, à condition qu'ils soient proches des usagers, permanents, et qu'ils aient un coût supportable par les ménages. 


\title{
ANALYSE DES SERVICES D'EAU NON CONVENTIONNELS DANS LES QUARTIERS À HABITAT SPONTANÉ : CAS DU QUARTIER EBA'A DANS LA PÉRIPHÉRIE DE LA VILLE DE YAOUNDÉ (RÉGION DU CENTRE AU CAMEROUN)
}

Analysis of non-conventional water services in spontaneous habitat districts: case study of the EBA'A District in the periphery of the Younde City (Center region in Cameroon)

\author{
BARTHELÉMY NDONGO ${ }^{1 *}$ EMMANUEL NGNIKAM², STÉPHANE LAKO MBOUENDEU ${ }^{3}$ \\ ${ }^{1}$ Département du Génie Rural, FASA, Université de Dschang, BP 222, Dschang, Cameroun. \\ ${ }^{2}$ Laboratoire de l'Environnement et des Sciences de l'Eau (LESEAU) ENSP, Université de Yaoundé I, \\ BP 3356, Yaoundé, Cameroun \\ ${ }^{3}$ Département du Génie Rural, FASA, Université de Dschang, BP 222, Dschang, Cameroun.
}

Reçu le 18 octobre 2010, accepté le 21 juin 2012

\section{RÉSUMÉ}

Cet article présente une analyse du service d'eau non conventionnel (service n'ayant fait l'objet d'aucune convention (cahier de charge, contrat, norme ou règlement, etc.), et donc organisé de façon intuitive par les utilisateurs) d'un quartier à habitat spontané de la périphérie de Yaoundé (EBA'A). L'étude aura consisté à : identifier les caractéristiques de ce service, relever les contraintes auxquelles ses usagers sont soumis, identifier les principaux déterminants de satisfaction de la clientèle et définir les perspectives d'amélioration de ce service. Pour cela, une enquête ménage a été effectuée dans le quartier sur un échantillon représentatif de la population étudiée. Cette enquête fut complétée par des observations et mesures faites sur le terrain. L'analyse des données collectées révèle que les services d'eau locaux non conventionnels s'appuient sur des ouvrages de desserte autonomes qui sont considérablement éloignés des usagers. Ce type de service semble contraignant pour les ménages d'EBA'A, et ce, plus sur le plan des conditions d'approvisionnement que du coût du service. C'est pourquoi, en guise d'amélioration, les ménages souhaitent avoir un service d'eau potable proche et permanent, même si son coût demeure élevé. Cependant, il existe un seuil de $2,10 \$ \bullet \mathrm{m}^{-3}$ pour ces populations. Dans ces conditions, un délai de paiement des factures d'au moins dix jours, avec des interruptions de service n'excédant pas trois jours, semble tolérable pour ces ménages. Ainsi, les services d'eau potable non conventionnels pourraient s'avérer être des alternatives d'accès à l'eau potable satisfaisantes dans les quartiers défavorisés, à condition qu'ils soient proches des usagers, permanents, et qu'ils aient un coût supportable par les ménages.

Mots clés : eau potable, service d'eau, gestion pérenne, prix de l'eau, habitat spontané, échantillonnage.

Auteur pour correspondance :

Téléphone: +23777564096/+23797917706

I+237222368 89

Courriel : bandongo@yahoo.fr 


\section{ABSTRACT}

An analysis of the informal water service of a spontaneous dwelling district in the periphery of Yaounde (EBA'A) was carried out. The work was to identify the features of the service, the difficulties encountered by its users, the main factors used to measure satisfaction of the clientele, and to define the perspectives for improvement of this service. In order to achieve this, a survey was carried out in the district on a representative sample of the population, together with field observations. Analysis revealed that the local water services depend on independent sources that are considerably far from the consumers. This type of service seems to be inconvenient for the households of EBA'A, more because of supply than the cost of the service. That's why, by way of improvement, the households would like a drinking water source that is nearby and permanent, even if the cost is high. However, there is a break-even point of $\$ 2.10 \cdot \mathrm{m}^{-3}$. In this case, a delay of payment of the invoices of at least ten days, with interruptions of service not exceeding three days, would be acceptable for these households. Non-conventional drinking water services would be acceptable alternatives to access potable water in spontaneous districts, if they were near, permanent and at acceptable cost.

\section{Key words: drinking water, water services, durable manage- ment, cost of water, spontaneous dwelling, sampling.}

\section{INTRODUCTION}

L'eau douce est une ressource rare et limitée sur la planète Terre. Selon les Nations Unies (UNWATER, 2009), 340 millions de personnes en Afrique n'ont pas accès à l'eau potable et trois millions d'entre elles meurent chaque année de maladies liées à une eau souillée. Au Cameroun, la proportion des ménages desservis par les réseaux d'eau conventionnels est en décroissance au fil des années, malgré les efforts de réhabilitation et de développement de ce service; 23,0 \% en 2004 (INONI, 2007) et 22,9 \% en 2007 (MINEE, 2008). Ceci serait dû à la réduction des investissements de l'État dans le secteur de l'amélioration de l'accès à l'eau potable (LESEAU, 2000), l'urbanisation galopante des grandes métropoles telle Yaoundé (DJEUDA et al., 2001), la complexité des solutions techniques et leur coût élevé qui ne justifient pas souvent l'investissement (LAKO, 2010).

Afin de soutenir les actions de l'État Camerounais, les organismes non gouvernementaux ont entrepris depuis bon nombre d'années de subventionner des actions d'amélioration de l'accès à l'eau potable. Celles-ci ont souvent connu une fin bien précoce. DJEUDA et al. (2001) expliquent cela par le fait que " la notion de service à l'usager n'est pas suffisamment intégrée dans les démarches qui sont expérimentées; les pratiques de l'usager sont mal connues et les schémas dans lesquels elles pourraient mieux s'intégrer ne sont pas suffisamment étudiés. ". En outre, seuls les projets d'amélioration de l'accès à l'eau potable qui ont bénéficié d'une bonne gestion, prenant en compte les facteurs sociaux et économiques locaux, parviennent à subsister (INFORESSOURCES, 2003). Un cas d'espèce est le projet SCANWATER qui a coûté 105,5 millions \$ au Cameroun pour la réalisation de stations de pompage et de traitement d'eau dans 365 localités en milieu semi-urbain et rural Ce projet SCANWATER, financé par la coopération danoise entre 1980 et 1991, avait pour but l'approvisionnement en eau potable d'environ 146000 personnes par 365 stations de pompage-traitement de l'eau sur toute l'étendue du territoire camerounais. Jusqu'en 1990, ces stations ont fait l'objet d'un suivi et d'un entretien encadré par l'État, où les populations (bénéficiaires) n'avaient aucune responsabilité au niveau des charges. Au cours des années 1990, l’État connait diverses crises financières qui le contraignent à suspendre le suivi et l'entretien des stations. Après quoi, la plupart d'entre elles vont tomber en panne pour être ensuite abandonnées par les populations. Un échec fatal sous le regard impuissant des bénéficiaires. Toutefois le gouvernement entreprend en 1996 la réhabilitation des stations susceptibles d'être viabilisées. Malgré cette action, des échecs ont encore été enregistrés. Par contre, on note que certaines stations installées depuis 1980 sont restées en fonctionnement de manière continue du simple fait de l'implication soutenue des populations (bénéficiaires) dans leur gestion. $80 \%$ de ces installations sont hors service malgré les réformes proposées par l'État au fil des années. Quelques-unes n'auraient survécu que grâce à l'émergence des associations d'usagers d'eau initiées par les populations ellesmêmes (DJEUDA et al. ,2001).

Le quartier EBA'A, malgré ses cinq décennies d'existence, est pratiquement exclu du service d'eau conventionnel urbain par sa position en altitude au-dessus de tous les réservoirs d'eau de Yaoundé, d'où son faible taux d'accès estimé en 2003 à 13,1 \% (ERA, 2003). Ainsi, pour satisfaire leurs besoins hydriques, les populations d'EBA'A ont développé un service d'eau local s'appuyant essentiellement sur des ouvrages hydrauliques autonomes. L'accès à l'eau potable figurant comme un problème prioritaire de la localité (ERA, 2003), il serait risqué ici, d'après TANAWA (2002), compte tenu de la complexité de la gestion des systèmes hydriques dans les pays en développement, de proposer des solutions conventionnelles sans réflexion, ni études préalables. Une amélioration durable de ce service demande dès lors de comprendre les processus d'urbanisation en cours et de rechercher les éléments de compréhension et de visibilité des processus qui conduiraient les individus et la collectivité à mettre en péril les systèmes hydriques améliorés alternatifs. Cette étude se propose par conséquent de contribuer à une meilleure connaissance du service d'eau local, pilier de la définition d'alternatives 
d'approvisionnement appropriées et viables, avec pour corollaire un accès durable des populations d'EBA'A à l'eau potable. De manière spécifique, cette étude s'attelle à caractériser le service d'eau local, à en identifier les contraintes, à déterminer les principaux paramètres de satisfaction de la clientèle ainsi que les perspectives d'amélioration.

\section{MATÉRIEL ET MÉTHODES}

\subsection{La zone d'étude}

La zone d'étude est le quartier EBA'A (présenté à la Figure 1), aussi connu sous le nom de Mvog-Betsi. C'est une localité du Cameroun dans la ville de Yaoundé. Elle se situe entre le $3^{\circ} 51^{\prime \prime} 19$ et $3^{\circ} 52^{\prime \prime} 00$ latitude Nord et s'étend de $11^{\circ} 28^{\prime} 09^{\prime \prime}$ à $11^{\circ} 29^{\prime} 20^{\prime \prime}$ longitude Est pour une superficie de $1,54 \mathrm{~km}^{2}$. Le quartier est divisé en neuf blocs. Cependant la zone d'intérêt se limite aux blocs 1, 2, 3, 4 et 9 avec une population de 5600 personnes (GMUS, 2008). Le climat est équatorial de type guinéen (SUCHEL, 1988), et le territoire est arrosé par deux cours d'eau : Biyeme et Mewoulou. L'altitude moyenne oscille entre 800 et $900 \mathrm{~m}$, largement au-dessus du plus haut réservoir de la ville (plateau ATEMENGUE) qui culmine à $750 \mathrm{~m}$ d'altitude (BIDALA, 2004). Par ailleurs, dans l'ensemble, la dotation du quartier en infrastructures et en services de base est faible (ERA, 2003). En effet, le réseau de l'ex-SNEC (Société Nationale des Eaux du Cameroun) privatisée en 2007, en affermage à la Camerounaise Des Eaux (CDE) est présent dans ce quartier, mais seul le bloc 1 dispose irrégulièrement de l'eau potable.

\subsection{La méthodologie}

Des entretiens et une enquête de terrain, effectués dans la période de juillet à octobre 2009, ont constitué les éléments fondamentaux de la méthodologie. L'enquête s'est appuyée sur des observations in situ et un questionnaire semistructuré, administré par la méthode d'entrevue qui portait sur 1083 ménages recensés dans la zone étudiée (ERA, 2007). Un échantillon représentatif de 118 ménages répartis sur toute la

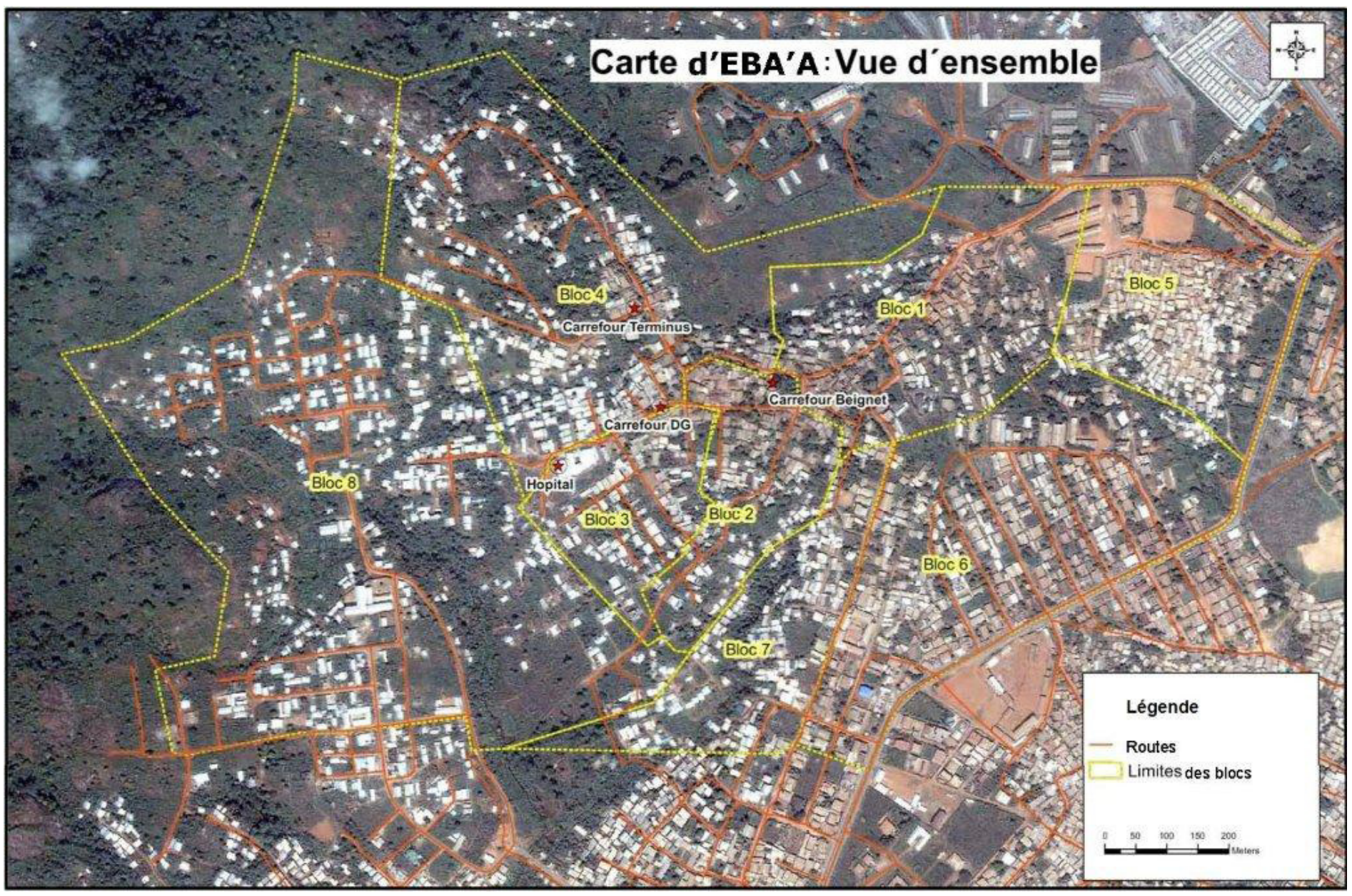

Figure 1. Vue aérienne du quartier EBA'A. Aerial view of the EBA'A District. 
zone d'intérêt a été retenu, soit un taux de sondage de 10,9\%, somme toute conforme à la norme statistique qui requiert au moins $10 \%$ (ISPEA, 1982).

\subsection{La taille de l'échantillon}

La taille de l'échantillon a été calculée par la méthode définie par OLIVERO (2001) pour la mesure des fréquences et résumée par l'équation 2.1 .

$$
\mathrm{N}=\mathrm{f}(1-\mathrm{f}) \times\left(\mathrm{U}_{1-\alpha / 2} / \mathrm{I}\right)^{2}
$$

où :

$\mathrm{U}_{1-\alpha / 2}$ : Coefficient de confiance;

I : $\quad$ Imprécision tolérée sur la fréquence (exprimée en \%);

$\mathrm{f}$ : Fréquence observée dans un échantillon de taille suffisante (effectif $>30$ ) et

$\alpha: \quad$ Niveau de risque.

Dans le cadre de l'étude, la fréquence observée est la fréquence des ménages enquêtés qui auraient sollicité une gestion communautaire.

Ainsi, pour des paramètres $\alpha=95 \%, \mathrm{I}=8 \%, \mathrm{f}=22 \%$, nous obtenons: $\mathrm{U}_{1-\alpha / 2}=1,96$ et $\mathrm{N}=103$ individus.

Cependant, pour tenir compte des questionnaires biaisés et des non-réponses, l'échantillon a été augmenté de $15 \%$, soit 118 ménages en tout. Ces ménages ont été répartis équitablement sur les cinq blocs $(1,2,3,4,9)$ renfermant la population étudiée.

\subsection{L'échantillonnage}

Le choix des ménages à enquêter a demandé l'usage de deux méthodes d'échantillonnage : la méthode des quotas et la méthode d'échantillonnage aléatoire systématique. La première aura permis de déterminer le nombre de ménages à enquêter par bloc et par sous-bloc (04 par bloc de superficie quasi identique). La deuxième aura servi à identifier les ménages à enquêter dans chaque sous-bloc. Il aura fallu pour cela recenser et classer les ménages de chaque sous-bloc par ordre croissant de numéro d'habitation. Le pas calculé sur la base des quotas de questionnaire par sous-bloc a permis de dégager une liste des ménages à enquêter. Le recensement des ménages aura été possible grâce à des photographies aériennes développées à l'aide du logiciel ArcGis (version 3.9).
Les critères de choix ont été le bloc de résidence et le statut d'occupation du ménage (locataire ou propriétaire) seulement pour les blocs 1 et 3, compte tenu des informations disponibles.

\subsection{Le questionnaire}

Le questionnaire soumis aux ménages des blocs du quartier EBA'A a été structuré en quatre sections, à savoir : 1) l'identification, 2) la gestion du service d'eau actuel et les exigences pour le service futur, (3) les dépenses liées à l'eau et (4) l'impact éventuel des améliorations du service sur la consommation d'eau.

Le questionnaire a été administré par la méthode d'entrevue et adressé aux responsables des ménages masculins ou féminins âgés d'au moins 21 ans. Cette phase a été précédée d'une préenquête qui a permis de clarifier les questions et de les formuler dans un style simple. Une formation des enquêteurs aura servi à l'harmonisation de la compréhension du questionnaire et la mise en œuvre d'un plan de couverture du quartier limitant au mieux l'imprégnation à l'avance des ménages qui auraient inséré un biais dans la qualité des réponses obtenues.

\subsection{Le traitement des informations collectées}

Le dépouillement des questionnaires s'est fait avec le concours des enquêteurs pour éviter la distorsion de l'information collectée et la répétition des enregistrements. Lélaboration d'un masque de saisie a permis la constitution de la base de données et l'analyse des résultats de l'enquête. L'utilitaire EXCEL de la suite Office 2003 a servi de logiciel de travail.

\subsection{L'estimation de l'éloignement}

La difficulté de l'estimation numérique du caractère d'éloignement des sources d'approvisionnement a conduit à évaluer ce paramètre à l'aide de quatre éléments d'appréciation courants : proche, pas très loin (distance tolérable), loin et très loin. Vu la difficulté de pouvoir mesurer (sur le terrain) cette distance pour chaque ménage enquêté, compte tenu de la multiplicité des trajets possibles, les ménages ont été regroupés sur une carte du quartier (échelle 1/5000) autour des points d'approvisionnement respectifs et la distance (à vol d'oiseau) correspondant au caractère d'éloignement fut déterminée. Les valeurs moyennes obtenues ont été pondérées par un coefficient tenant compte de l'effet de contournement et du coefficient moyen de pente pour le trajet réel le plus court. Pour l'effet de contournement, les distances ont effectivement été parcourues 
et évaluées. La carte topographique de la zone a permis, quant à elle, d'intégrer l'effet de la dénivelée. Toutefois, l'impact sur le coût réel d'acquisition de l'eau n'a pu être évalué, compte tenu de la multiplicité des moyens de transport (à pied, à moto ou dans de rares cas en voiture).

\subsection{L'estimation du temps moyen de puisage}

Les sources d'approvisionnement en eau potable les plus utilisées ont été identifiées. Par la suite, des usagers choisis au hasard sont suivis afin de déterminer la durée nécessaire pour la collecte de l'eau à chaque point de desserte. Les quantités collectées sont relevées et associées aux durées réelles pour établir une durée équivalente à un standard de $10 \mathrm{~L}$, par souci de comparabilité des données. Cette opération aura été répétée cinq fois pour chaque point d'eau afin de limiter les erreurs. Une évaluation des débits par la méthode volumétrique permet dès lors de déterminer la durée théorique de puisage de $10 \mathrm{~L}$ d'eau.

\subsection{La détermination des critères principaux de satisfaction des usagers du service}

La motivation exprimée du caractère de satisfaction ou non du service d'eau local aura permis une analyse des principaux déterminants de la satisfaction de la clientèle d'EBA'A. L'étude s'est intéressée à quatre facteurs qui ont été : l'accès direct au service CDE (Camerounaise Des Eaux), l'accès antérieur au service conventionnel SNEC (Société Nationale des Eaux du Cameroun), la proximité et la gratuité du service d'eau. Une analyse des proportions de ménages concernés par l'un ou l'autre facteur aura indiqué les principaux déterminants de la satisfaction chez les ménages de la zone d'étude.

\section{RÉSULTATS ET DISCUSSION}

\subsection{Caractérisation du service d'eau local}

\subsubsection{Ouvrages d'approvisionnement}

Les populations de la zone d'étude disposent de deux catégories de source d'approvisionnement en eau : les ouvrages d'approvisionnement communautaires (trois sources, deux puits, un forage et deux bornes-fontaines) et les points d'approvisionnement privés (puits à domicile et connexions CDE à domicile). Comme il a été souligné par Le BRIS (2000), les ménages d'EBA'A sont attachés à la complémentarité des modes d'alimentation et les sources sollicitées pour la boisson sont, par ordre de préférence, l'eau du réseau et des sources d'eau aménagées.
Cependant, le service d'eau local s'appuie sur quatre types d'ouvrages.

\subsubsection{Trois sources sommairement aménagées}

Il s'agit de la source en face de la chefferie à l'entrée du quartier (Bloc 1), la source Mokala au Sud-Est et la source Mewoulou au Nord-Ouest. Elles disposent chacune d'un seul point de puisage. La rampe de puisage n'est pas aménagée et la distance du tuyau de puisage par rapport à la surface de l'eau sur la rampe n'excède pas $40 \mathrm{~cm}$, ce qui expose l'eau puisée aux souillures provoquées par l'effet éclaboussure. Par ailleurs, bien que la chambre de captage soit dallée, la proximité des habitations est une règle générale dont seule la source Mewoulou fait exception.

\subsubsection{Deux puits communautaires}

Le premier puits est situé au centre du quartier et l'autre au sud. Les deux sont dotés d'une plateforme dallée surmontée d'une superstructure circulaire et rectangulaire respectivement de $60 \mathrm{~cm}$ de haut chacune. L'ouverture de puisage a une surface inférieure de $0,2 \mathrm{~m}^{2}$. Le premier puits est entouré dans un rayon de $10 \mathrm{~m}$ par les habitations, tandis que le second est en zone d'altitude basse marécageuse. Le niveau statique de l'eau est de $2 \mathrm{~m}$ au plus pour les deux puits et la zone de captage est la nappe phréatique.

\subsubsection{Un forage communautaire}

Il est construit sous abri à l'ouest du quartier et doté d'une pompe manuelle de marque "India Mark II » reposant sur un socle en béton. L'espace de puisage est muni de pentes autocurantes sur ses quatre faces qui conduisent les eaux dans les caniveaux alentours.

\subsubsection{Deux bornes-fontaines payantes}

De ces bornes-fontaines, seule une sert à l'usage public. Cette dernière se résume à une canalisation en acier galvanisé recouverte de rouille, élevée à $1 \mathrm{~m}$ du sol et maintenue par un tuteur en bois.

\subsubsection{Modes de paiement de l'eau}

Les modes d'accès à l'eau dans le quartier sont de deux types : les modes gratuits et les modes payants qui desservent respectivement 28 et $69 \%$ des ménages. Certes la population semble majoritairement habituée à un service payant mais il convient de relever que l'eau achetée sert beaucoup plus à la boisson et à la cuisson, quelques fois à la vaisselle et au bain.

\subsubsection{Le paiement par forfait}

Il concerne particulièrement les points d'approvisionnement privés. L'accès à l'eau CDE se fait à des forfaits variant entre 2,10 et $4,20 \$ /$ mois, alors qu'au puits, les usagers paient entre 1,24 et $2,72 \$ /$ mois. Les frais payés pour le puits couvrent l'abonnement, l'achat du matériel d'exhaure et l'entretien de l'ouvrage. 


\subsubsection{Le paiement au volume}

Il porte essentiellement sur l'eau fournie par la CDE. Il se fait aussi bien aux bornes-fontaines payantes, au forage, que chez des particuliers. Les prix pratiqués sont de : trois bouteilles de 1,5 L à 2 cents; 2 à 3 cents le seau de $10 \mathrm{~L}$, et 4 à 10 cents le bidon de $20 \mathrm{~L}$. Cette variabilité est liée à la volonté des commerçants qui fixent leurs prix en fonction de la proximité des usagers, de la qualité « relative » de l'eau et de sa disponibilité.

Le service aux bornes-fontaines payantes et au forage se fait entre $6 \mathrm{~h}$ et $8 \mathrm{~h}$ et $16 \mathrm{~h}$ et $19 \mathrm{~h}$ (les heures sont données en référence à l'heure locale $(\mathrm{GMT}+1))$. En saison sèche, il s'étend de $4 \mathrm{~h}$ à $23 \mathrm{~h}$. Ces horaires valent aussi pour les ouvrages de service privés fonctionnant avec un mode payant. Pour les ouvrages privés de mode gratuit, l'accès est libre autant que dans les sources et les puits communautaires. Cependant, en saison sèche, les habitants sont obligés de sortir de leur couche à $2 \mathrm{~h}$ du matin pour espérer puiser de l'eau à $6 \mathrm{~h}$. Ceci est dû au grand nombre d'usagers et à la réduction drastique des débits des sources pendant cette période de l'année.

\subsection{Les contraintes du service d'eau local}

\subsubsection{Léloignement des points de desserte}

L'éloignement concerne la distance séparant les ménages de leur source d'approvisionnement en eau de boisson. L'analyse de la figure 2 nous montre que : $17 \%$ des ménages sont situés à $50 \mathrm{~m}$ au plus de leur source d'approvisionnement en eau de boisson, tandis que $54 \%$ parcourent au moins $151 \mathrm{~m}$ pour s'approvisionner.

Ces distances ne témoignent pas toujours de l'effort fourni par les usagers car l'appréciation dépend des contextes d'approvisionnement et des moyens humains, temporels et financiers. Ceci s'observe dans l'ambiguïté que présentent les caractères " loin » et " très loin " au tableau 1. Globalement, $28 \%$ des ménages pensent être proches de leur source d'approvisionnement, $23 \%$ estiment la distance acceptable, tandis que $49 \%$ se sentent assez éloignés du lieu d'approvisionnement. Cet éloignement a pour corollaire, soit l'accentuation de la corvée d'eau, soit un surcoût lié au transport de l'eau (par moto ou taxi). Par ailleurs, le tableau 1 montre que les sources et le forage sont moins éloignés des consommateurs que les points de distribution d'eau $\mathrm{CDE}$, ce qui souligne le niveau de compétitivité élevé de ces sources d'eau. JANIQUE et al. (1998) font d'ailleurs remarquer que : "La concurrence des sources d'approvisionnement d'eau gratuites peut mettre en péril la rentabilité financière et les bénéfices sanitaires de points d'eau collectifs payants dont l'usage ne s'impose pas d'emblée ».

Dans le contexte d'EBA'A, l'effet de la topographie et du contournement peut contribuer à tripler la distance à parcourir

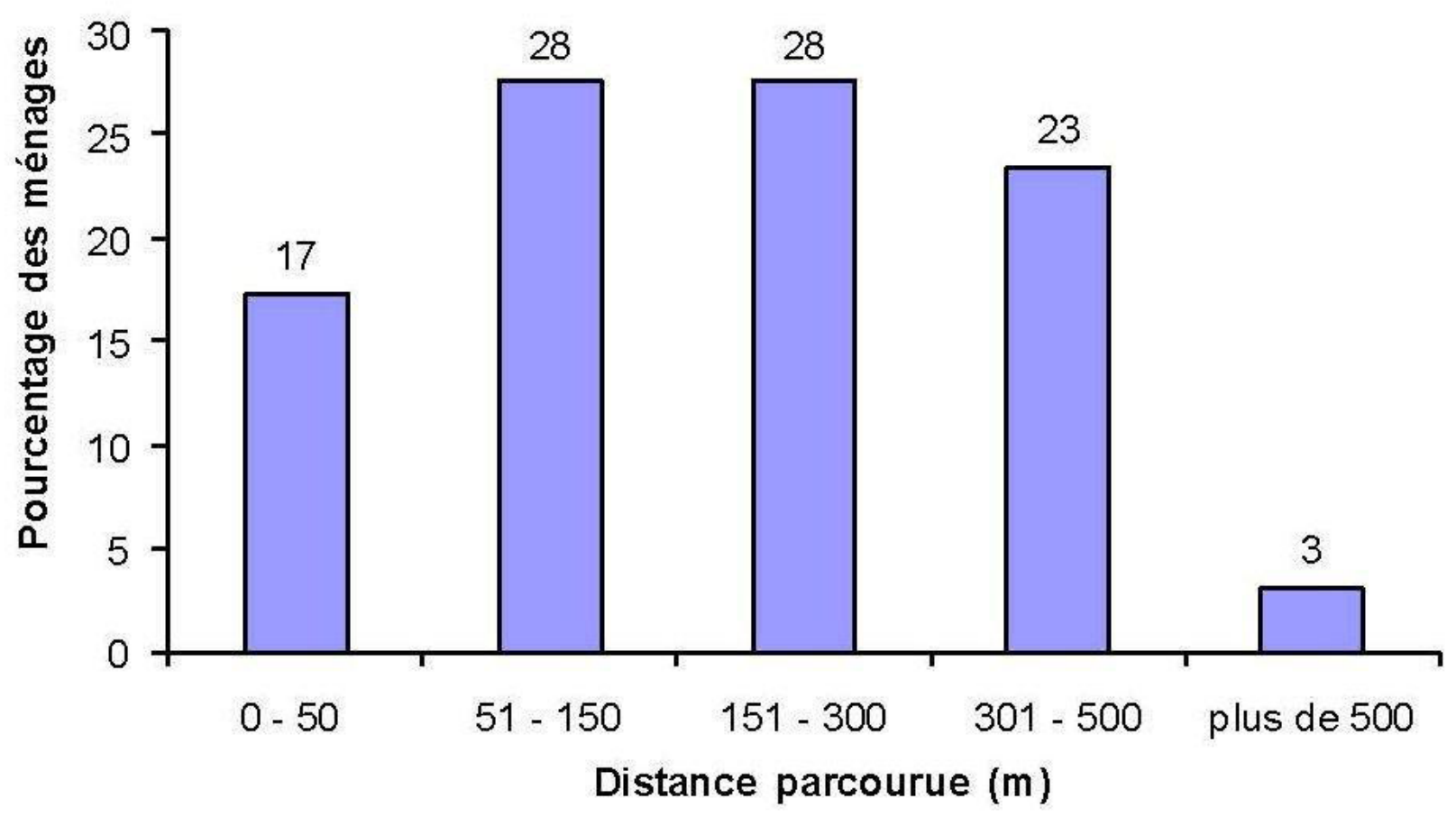

Figure 2. Distance parcourue par les ménages pour s'approvisionner en eau de boisson. Distance covered by the households to get drinking water. 
Tableau 1. Perception des ménages de leur éloignement selon l'ouvrage d'approvisionnement.

Table 1. Perception of the households of their remoteness relative to the provision source.

\begin{tabular}{lcccc}
\hline Éloignement & Proche & Pas très loin & Loin & Très loin \\
Distance réelle $(\mathrm{m})$ & 56 & 190 & 956 & 415 \\
$\begin{array}{l}\text { Coefficient de correction } \\
\text { de la distance théorique }\end{array}$ & 1,1 & 1,2 & 2,9 & 1,4 \\
\hline CDE (\%) & 24 & 13 & 16 & 13 \\
Source (\%) & 4 & 8 & 10 & 8 \\
Forage (\%) & 0 & 2 & 0 & 1 \\
Eau minérale (\%) & 0 & 0 & 0 & 1 \\
Total & 28 & 23 & 26 & 23 \\
\hline
\end{tabular}

pour atteindre le point d'eau. La frontière d'éloignement estimée, en moyenne à $190 \mathrm{~m}$, semble être une valeur intermédiaire correspondant aux zones péri-urbaines, car MOREL A L'HUSSIER, cité dans JANIQUE et al. (1998), a montré que, dans certains pays d'Afrique, le seuil de distance entre la proximité et l'éloignement perçu est de $120 \mathrm{~m}$ dans les quartiers urbains et de $250 \mathrm{~m}$ dans les zones rurales. Il apparaît donc primordial que les programmes d'hydraulique tiennent compte du facteur éloignement pour chaque contexte (pénibilité du transport) dans la répartition des ouvrages d'approvisionnement en eau potable.

\subsubsection{La satisfaction de la demande en eau}

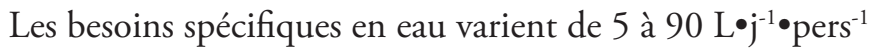
avec une moyenne de $23 \mathrm{~L}^{\circ} \mathbf{j}^{-1} \bullet$ pers $^{-1}$, inférieure aux $30 \mathrm{~L}^{\circ} \mathbf{j}^{-1} \bullet$ pers $^{-1}$ préconisés par la DIEPA (Décennie Internationale de l'Eau Potable et l'Assainissement, décrétée par l'assemblée générale des Nations Unies le 11 novembre 1980). La consommation spécifique moyenne correspond dès lors à un service minimum exigible de $253 \mathrm{~m}^{3} \bullet^{-1}$ pour une population estimée à l'échéance 2018 à 10029 habitants : une demande qui est largement au-dessus de l'offre; $36 \mathrm{~m}^{3} \bullet \mathrm{j}^{-1}$ produits par l'ensemble des ouvrages du quartier (par voie des sources pseudo-aménagées, forages et bornes-fontaines), d'où le recours aux sources d'eau à risque (puits, rivières, pluies). Du fait de la croissance démographique dans le quartier et la stagnation du nombre de branchements au réseau conventionnel, le taux d'accès direct à l'eau CDE dans la zone étudiée s'amenuise rapidement. Il est passé de $13 \%$ en 2003 (ERA, 2003) à $10 \%$ en 2009 (LAKO, 2010), ce qui risque de compromettre l'atteinte des OMD en matière d'accès à l'eau potable en 2015 dans cette localité. Ce faible taux est compensé par la revente de l'eau qui assure un accès indirect à $58 \%$ des ménage,; cette eau étant essentiellement utilisée pour la boisson. Le tableau 2 montre en effet que $61 \%$ des ménages utilisent l'eau CDE pour la boisson.

L'examen de ce tableau indique que l'eau de la CDE et l'eau de source sont plus utilisées pour la consommation (boisson et cuisson), tandis que l'eau de puits n'est pas utilisée pour la boisson mais se présente comme la première source d'eau de ménage. Les eaux de profondeur (forage, eau minérale) sont exclusivement utilisées pour la consommation. Cela traduit la complémentarité des modes d'approvisionnement que Le BRIS (2000) estime être caractéristique des pratiques propres aux quartiers pauvres.

\subsubsection{Le prix élevé du service}

D'après la figure 3 , les prix de l'eau varient de 0 à $5,3 \$ \bullet \mathrm{m}^{-3}$, la moyenne étant de $1,8 \$ \bullet \mathrm{m}^{-3}$ largement au-delà du prix pratiqué par les services conventionnels CDE au Cameroun $\left(0,8 \$ \bullet \mathrm{m}^{-3}\right)$. L'analyse de cette figure mène au constat que $30 \%$ des ménages ne paient rien pour l'eau tandis que $7 \%$ paient plus de $4,2 \$ \bullet \mathrm{m}^{-3}$. En outre, les ménages bénéficiant d'un service gratuit sont pour la plupart des abonnés des sources et des puits.

La même figure indique que la moyenne de prix tolérable par les ménages est de $1,4 \$ \bullet \mathrm{m}^{-3}$. Ce tarif est inférieur au prix moyen pratiqué actuellement $\left(1,8 \$ \bullet \mathrm{m}^{-3}\right)$. Il apparaît ainsi que les populations souhaiteraient payer l'eau moins cher. Cependant, conformément à l'observation de VINCENT (2003) selon laquelle : "les communautés urbaines et rurales des pays en développement étaient prêtes à payer un prix supérieur à celui des tarifs actuels des systèmes collectifs d'approvisionnement afin d'obtenir un service de meilleure qualité ou plus fiable", $84 \%$ des ménages d'EBA'A sont disposés à payer entre 0 et $2,1 \$ \cdot \mathrm{m}^{-3}$. 
Tableau 2. Pourcentages des ménages consommant de l'eau selon sa nature et son usage.

Table 2. Percentages of the households consuming water according to its nature and its use.

\begin{tabular}{|c|c|c|c|c|c|c|c|c|c|}
\hline Usages & $\mathrm{CDE}$ & $\begin{array}{l}\mathrm{CDE}^{*} \\
\text { Forage }\end{array}$ & $\begin{array}{c}\text { CDE * } \\
\text { Puits }\end{array}$ & $\begin{array}{l}\mathrm{CDE}^{*} \\
\text { Source }\end{array}$ & $\begin{array}{c}\text { Eau * } \\
\text { Minérale }\end{array}$ & Forage & Puits & $\begin{array}{l}\text { Puits * } \\
\text { Source }\end{array}$ & Source \\
\hline Eau de boisson & 61 & 0 & 0 & 6 & 1 & 3 & 0 & 0 & 29 \\
\hline Eau de cuisson & 34 & 1 & 2 & 4 & 0 & 0 & 40 & 1 & 18 \\
\hline Eau de ménage & 22 & 0 & 2 & 5 & 0 & 0 & 52 & 2 & 17 \\
\hline
\end{tabular}

* Association des eaux de diverses origines.

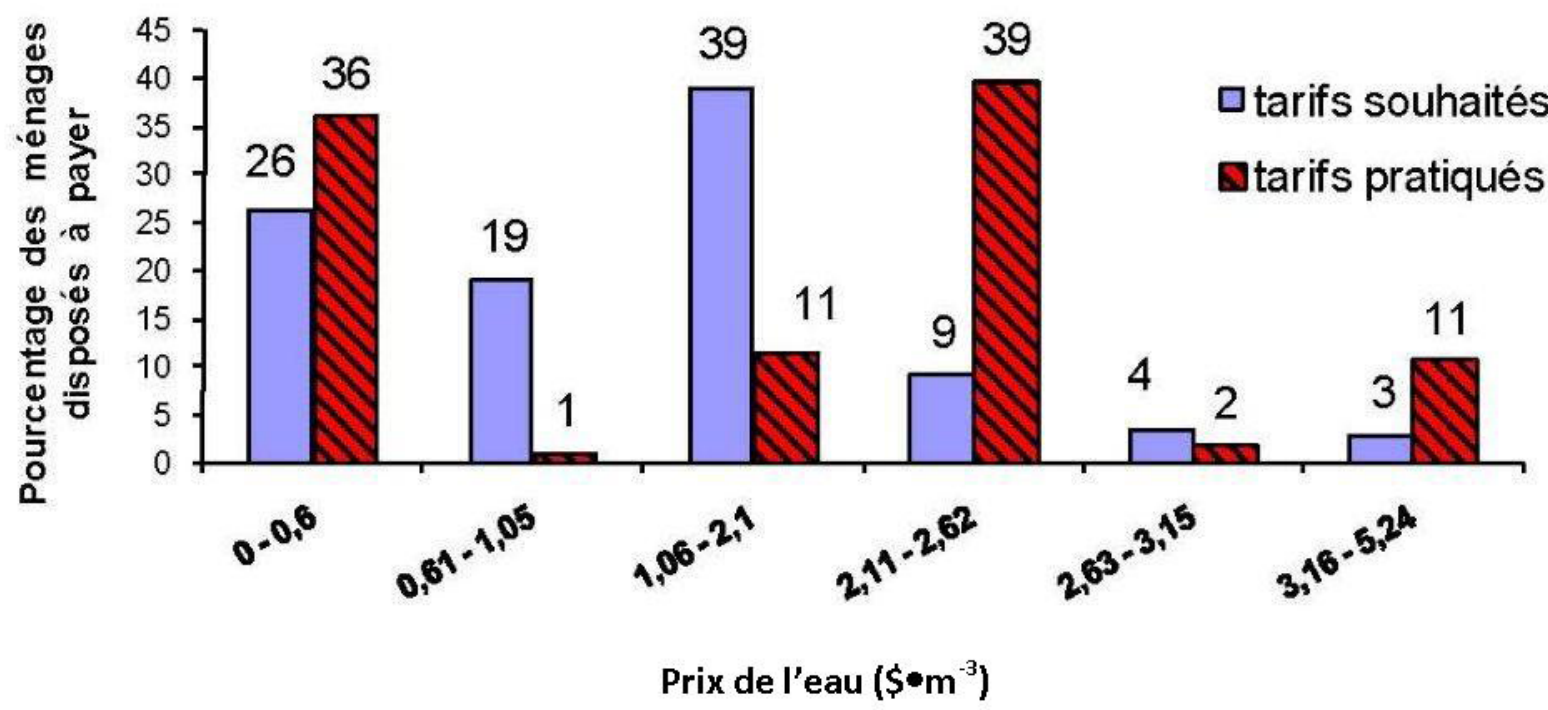

Figure 3. Disposition des ménages à payer un prix donné pour un service amélioré comparé aux prix pratiqués (hachuré). Willingness of households to pay a given price for an improved service compared to the current prices (hatched).

Toutefois, il se dégage que, contrairement à la pratique actuelle où $52 \%$ des ménages paient au moins $2,1 \$ \bullet \mathrm{m}^{-3}$, seuls $16 \%$ des ménages seraient disposés à payer un tarif compris entre 2,1 et $5,3 \$ \bullet \mathrm{m}^{-3}$. Ainsi, le seuil d'effort acceptable par les ménages d'EBA'A est en moyenne de $2,1 \$ \bullet \mathrm{m}^{-3}$.

\subsubsection{Le temps de puisage long}

Il s'étale de 23 à 86 minutes en moyenne, bien au-dessus du seuil défini par JANIQUE et al. (1998) à 20 minutes. Pour un standard de dix litres d'eau à collecter, les populations d'EBA'A passent entre 3 et 28 minutes au point d'eau, comme il est présenté au tableau 3. Le service à la borne-fontaine est le plus rapide du fait de son débit, la pression sur le service y est moindre à cause de son coût non nul. Bien que le rapport de déperdition de temps au point d'eau soit moins élevé chez ce dernier comparé aux sources, la durée réelle de collecte de l'eau est d'environ quatre fois la durée théorique (elle représente le temps nécessaire pour puiser de l'eau, tandis que la durée réelle est le temps total mis au point d'eau, incluant le temps d'attente et de nettoyage des contenants). Cet écart s'explique (en général) par l'addition, au temps effectif de puisage, du temps d'attente pour le service et de celui de manipulation des récipients (manutention, rinçage, etc.).

\subsection{Les principaux déterminants de la satisfaction des usagers $d u$ service d'eau}

Le tableau 4 donne une idée de l'influence des facteurs suivants sur la satisfaction des usagers du service : l'accès direct à l'eau, l'accès antérieur à un service satisfaisant, la proximité et la gratuité du service. 
Tableau 3. Temps de collecte de l'eau par type d'ouvrage de service.

Table 3. Time of water collection by type of service point.

\begin{tabular}{|c|c|c|c|c|}
\hline & Source Bloc 1 & $\begin{array}{c}\text { Source } \\
\text { Mewoulou }\end{array}$ & $\begin{array}{l}\text { Source } \\
\text { Mokala }\end{array}$ & Borne fontaine \\
\hline Durée de puisage équivalent $10 \mathrm{~L}$ (min) & 18 & 28 & 25 & 4 \\
\hline $\begin{array}{l}\text { Durée théorique de puisage (min) } \\
\left(10^{*} \mathrm{Q}\right)\end{array}$ & 2 & 2 & 4 & 1 \\
\hline Rapport temporel & 9 & 14 & 6,2 & 4 \\
\hline
\end{tabular}

Tableau 4. Perception des ménages selon le facteur de satisfaction.

Table 4. Perception of the households according to the satisfaction factor.

\begin{tabular}{|c|c|c|c|c|}
\hline $\begin{array}{l}\text { Appréciation du } \\
\text { service }\end{array}$ & Accès direct & Accès SNEC antérieur & Proximité & Gratuite \\
\hline Satisfait ((\%) & 36 & 64 & 59 & 23 \\
\hline Insatisfait (\%) & 4 & 63 & 20 & 31 \\
\hline Indécis (\%) & 20 & 80 & 40 & 20 \\
\hline
\end{tabular}

Le tableau 4 montre que $36 \%$ des ménages satisfaits par le service d'eau actuel dans la zone d'étude ont un accès direct à l'eau CDE et $59 \%$ de ceux-ci estiment avoir un service de proximité. Par ailleurs, $63 \%$ des ménages insatisfaits ont bénéficié dans un contexte antérieur d'un service SNEC (assimilable au service CDE). Cependant, 80 \% d'entre eux estiment ne pas être proches de leur source d'approvisionnement actuelle et seuls $4 \%$ d'entre eux ont actuellement un accès direct à l'eau CDE. Ainsi, plus les usagers ont accès directement à l'eau ou ont un accès indirect proche et de bonne qualité, plus ils sont satisfaits. En outre, la gratuité du service ne semble pas avoir d'influence notable sur la satisfaction des usagers, contrairement à l'observation faite par TAISNE (1997) dans la commune de Yaoundé IV ${ }^{e}$, ce qui s'explique par les conditions difficiles d'accès à l'eau qui occultent l'éventuel problème du coût. Ainsi les principaux éléments de satisfaction des ménages semblent être la proximité du service d'eau et sa qualité.

\subsection{Les perspectives d'amélioration du service d'eau}

L'analyse de la figure 4 montre que la principale amélioration attendue est un service de proximité (à proximité ou à domicile) qui soit permanent. Cependant, $2 \%$ des ménages pensent qu'une réfection des ouvrages d'approvisionnement actuels suffirait à améliorer de façon satisfaisante le service d'eau. En effet, $96 \%$ des ménages ont déjà eu accès à un service d'eau proche et de qualité satisfaisante (SNEC), d'où la tendance à considérer la proximité du service et sa qualité comme premières améliorations sollicitées par les populations d'EBA'A.

Par ailleurs, la baisse du prix du service semble ne pas être la priorité; ceci pourrait se justifier par la rareté actuelle de l'eau potable qui leur impose de se contenter de ce qu'ils ont.

Dans l'hypothèse d'un service évoluant vers le service conventionnel CDE, les ménages déclarent être favorables à un délai de paiement variant entre 5 et 30 jours. Cependant, la majorité des ménages $(80 \%)$ préfere un délai au moins égal à dix jours. Ceci correspond au délai accordé pour le paiement des factures d'eau et d'électricité au Cameroun. En effet, les ménages bénéficient d'un service d'électricité offrant un délai de paiement de dix jours qui semble satisfaisant. Notons qu'au Cameroun, la facturation est faite sur la base des consommations; des relevés mensuels des compteurs volumétriques sont faits et les factures établies sur la base d'une tarification standard $\left(0,8 \$ \bullet \mathrm{m}^{-3}\right)$. Lorsque les factures sont distribuées, les consommateurs ont un délai de dix jours pour les payer, sans quoi le service leur est suspendu. Le délai de paiement est donc de dix jours.

L'accent mis sur la permanence du service relève également de la durée tolérable des interruptions (selon les causes) présentée au tableau 5. Les ménages sont globalement tolérants (80\%) à une interruption du service à condition que celle-ci ne dépasse pas trois jours pour la maintenance et deux jours pour 


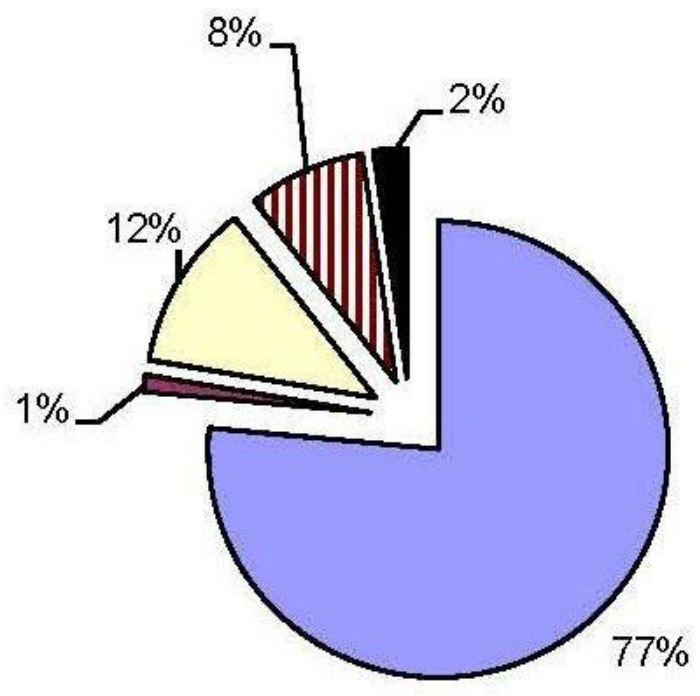

口Eau à domicile

Eau à domicile et baisse des prix

Eau à proximité

mEau en permanence

Réfection des installations

Figure 4. Proportions des ménages selon le type d'amélioration du service d'eau.

Proportions of the households according to the type of improvement of the water service.

Tableau 5. Pourcentages de ménages favorables à une durée d'interruption donnée.

Table 5. Percentages of households favourable to a given length of interruption.

\begin{tabular}{lccccccc}
\hline Délai (jours) & 0 & 1 & 2 & 3 & 5 & 7 & 14 \\
\hline Réparation & 1 & 37 & 37 & 12 & 1 & 10 & 2 \\
$\begin{array}{l}\text { Rétablissement } \\
\text { (après suspension) }\end{array}$ & 2 & 54 & 33 & 4 & 2 & 3 & 2 \\
\hline
\end{tabular}

le rétablissement, étant entendu qu'une interruption entraîne le recours à des sources d'eau moins fiables sur le plan sanitaire, exposant les consommateurs aux maladies d'origine hydrique et donc à des dépenses de santé supplémentaires.

\section{CONCLUSION}

L'analyse du service d'eau non conventionnel du quartier EBA'A, dans la périphérie de Yaoundé au Cameroun, nous a permis de constater que ce type de service s'appuie sur des ouvrages considérablement distants des consommateurs et offrant des débits globalement insuffisants. Les distances à parcourir et le temps nécessaire à la collecte de l'eau se présentent, dès lors, comme les principaux handicaps. Dans ces conditions, les ménages à faibles revenus ou à revenus instables s'orientent vers des usages des eaux d'origines diverses en fonction de leur accessibilité d'abord, leur disponibilité ensuite et enfin leur coût. Ainsi les perspectives d'amélioration d'après les ménages devraient s'orienter vers le rapprochement des points de desserte et la continuité du service même si le coût paraît élevé. Par conséquent, ces ménages sont disposés à payer plus cher un service amélioré s'ils ont l'assurance d'avoir un service de proximité, permanent et de qualité au moins comparable aux standards conventionnels nationaux. Cependant, il existe un seuil de coût au-delà duquel un tel service n'est plus attrayant, $2,1 \$ \bullet \mathrm{m}^{-3}$ dans ce cas. En définitive, les services d'eau non conventionnels se présentent comme une alternative exploitable d'amélioration de l'accès à l'eau potable dans les grandes villes du Cameroun où la crise de l'eau se fait de plus en plus pressante sous réserve des améliorations susévoquées. 


\section{RÉFÉRENCES BIBLIOGRAPHIQUES}

BIDALA A. (2004). Étude d'avant-projet sommaire d'un miniréseau d'adduction d'eau à EBA'A. ERA-CAMEROUN, Yaoundé, Cameroun, 45 p.

DJEUDA T.H.B., E. TANAWA, E. NGNIKAM (2001). L'eau au Cameroun, tome 1 : Approvisionnement en eau potable. Presses universitaires de Yaoundé, Yaoundé, Cameroun, $359 \mathrm{p}$.

ERA (Environnement, Recherche et Action au Cameroun) (2003). Rapport final d'exécution du projet Quartier. ERACAMEROUN, Yaoundé, Cameroun, 52 p.

ERA (Environnement, Recherche et Action au Cameroun) (2007). Rapport final du recensement de la population du quartier EBA'A. ERA-CAMEROUN, Yaoundé, Cameroun.

GMUS (2008). Avant-projet détaillé du mini-réseau d'eau d'EBA'A. Rapport mémoire technique. GMUS, Yaoundé, Cameroun, $26 \mathrm{p}$.

INFORESSOURCES (2003). Gestion intégrée des ressources en eau (GIRE) : la voie du développement durable. Focus $\mathrm{N}^{\circ} 01$, Zollikofen, Allemagne, $16 \mathrm{p}$.

INONI E. (2007). Lettre de politique sectorielle de l'hydraulique urbaine. Primature, Yaoundé, Cameroun, 7 p.

ISPEA (1982). Enquête par sondage sur le logement à Yaoundé. ISPEA, Yaoundé, Cameroun, $52 \mathrm{p}$.

JANIQUE E., H. COING, H. CONAN, S. JAGLIN, A. MOREL A L'HUISSIER, M. TAMIATTO et Y. VAILLEUX (1998). Analyse comparative de divers systèmes de gestion déléguée des points d'eau collectifs. Programme "Eau potable et assainissement dans les quartiers périurbains et les petits centres ", PSEAU (Programme Solidarité Eau), Paris, $127 \mathrm{p}$.

LAKO M.S. (2010). Contribution à la gestion pérenne du service d'eau dans les quartiers à habitat spontané : cas du quartier EBA'A (Yaoundé). Mémoire d'Ingénieur, Faculté d'Agronomie et des Sciences Agricoles, Université de Dschang, Dschang, Cameroun, 69 p.

LE BRIS E. (2000). Accès à l'eau potable dans les quartiers défavorisés des grandes villes et les petits centres urbains. Institut de Recherche pour le Développement (IRD), 14 p.

LESEAU (Laboratoire de l'Environnement et des Sciences de l'Eau) (2000). Les processus de décentralisation et leurs incidences sur les services sociaux de santé et d'éducation.
École Nationale Supérieure Polytechnique de Yaoundé, Université de Yaoundé I, Yaoundé, Cameroun, 124 p.

MINEE (Ministère de l'Énergie et de l'Eau) (2008). Amélioration de la fourniture en eau potable. Point de presse du Ministre de l'Énergie et de l'Eau sur le secteur de l'hydraulique, de l'hydrologie et de l'assainissement. MINEE, 18 décembre 2008, Yaoundé, Cameroun, rapport, 57 p.

OLIVERO P. (2001). Gestion des déplacements, évaluation d'impacts et tests de matériels: calcul de la taille des échantillons (application aux cas simples et usuels). CETE du Sud-Ouest, ZELT, Toulouse, France, 43 p.

SUCHEL, J.B. (1988). Les climats du Cameroun. Thèse de Doctorat d'État, Université Saint-Etienne, France, 1188 p.

TAISNE R. (1997). Réhabilitation de points d'eau populaires dans une grande métropole: Yaoundé, Cameroun. HYDRO CONSEIL, France, 75 p.

TANAWA E. (2002). La gestion durable des systèmes hydriques dans les pays en développement. Dans : Gestion intégrée de l'eau en Haïti. EMMANUEL, E. et P. VERMANDE(Éditeurs). Actes du colloque international de Port-au-Prince, 26 au 28 juin 2002, Villeurbanne, Haïti, 310 p.

UNWATER (Organisation des Nations Unies pour l'Eau) (2009). Statistics: Drinking water and sanitation. http:// www.unwater.org/statistics_san.html. (Consultation le 15 avril 2009).

VINCENT I. (2003). Le prix de l'eau pour les pauvres : comment concilier droit d'accès et paiement d'un service? Afrique contemporaine, 205, France, 16 p. 